\title{
Ex vivo performance evaluation of a transdermal gold injectable microneedle for tyrosinase sensing
}

\author{
Sorour Darvishi, ${ }^{[a]}$ Hubert H. Girault, ${ }^{*}[a]$ \\ [a] Laboratory of Physical and Analytical Electrochemistry, Ecole Polytechnique Fédérale de Lausanne \\ (EPFL) Valais Wallis, Rue de I'Industrie 17, 1950 Sion, Switzerland
}

\footnotetext{
* Corresponding author: Laboratory of Physical and Analytical Electrochemistry, Ecole Polytechnique Fédérale de Lausanne (EPFL) Valais Wallis, Rue de l'Industrie 17, 1950 Sion, Switzerland. Email addresses: hubert.girault@epfl.ch (H.H. Girault)
} 


\begin{abstract}
Here, the semi-invasive direct electrochemical detection of melanoma biomarkers in nontreated skin has been envisaged. The enzyme tyrosinase was to be addressed with a microneedle electrochemical sensor. The microneedles were fabricated by polydimethylsiloxane (PDMS) casting with stable polymers. The as-prepared microneedles (MNs) were then coated by gold sputtering. The gold MNs were finally covered by alginate/catechol to provide a liquid electrolyte layer that contained electroactive species, such as catechol, whose redox state can be linked to the concentration of TYR in the skin. The sensor showed high sensitivity of $7.52 \mu \mathrm{A} \cdot \mathrm{mg}^{-1} \cdot \mathrm{mL}$ TYR in dummy skin. A relative standard deviation (RSD) of $8.54 \%(n=5)$ demonstrated that the Catechol@alginate: gold MN electrode had an excellent reproducibility for continuous glucose detection. Also, five parallel Catechol@alginate: gold MN electrodes were fabricated using the same experimental setup and showed an RSD of $3.95 \%(n=5)$. These results suggest that the electrode fabrication process was reproducible, and Catechol@alginate: gold MN biosensors demonstrated high stability for the repetitive detection of TYR. Furthermore, the sensor has high selectivity in the presence of interfering components towards TYR screening.
\end{abstract}

Keywords: Electrochemical biosensor, Melanoma, Microneedle sensor, Tyrosinase screening, Catechol. 


\section{Introduction}

Diagnostic devices, including dermal biosensors, are becoming increasingly popular due to their high potential in the laboratory and their potential for personal health observations. Dermal biosensors, i.e., sensors applied to detect analytes selectively in the dermis, are divided into two main groups: wearable sensors, including skin-worn surface electrodes, and needle-type skin-penetrable biosensors. These classes of devices were broadly developed to analyze a wide range of analytes in skin tissue and fluids, including sweat [1] and interstitial fluid (ISF) [2], to detect biomarkers for diseases and cancer, such as melanoma [3]. Among these two classes of skin sensors, skin-penetrating microneedle array biosensors are considered one of the most promising skin-diagnostic devices. At the same time, and if welldesigned in terms of materials and geometries, they enable access to the dermal layers without or only with little causing pain or bleeding while providing robust information [4]. Apart from the potentially obtained pain or bleeding, penetrating needles should not provoke the spread of for instance cancer cells to other body parts when hitting blood vessels.

The sensitive and reliable detection of tumor markers, particularly at the early stage of cancer, is currently the subject of many intensive studies, particularly melanoma. In general, a wide range of label-free immunosensing methods has been reported for the detection of cancers, which includes the use of optical techniques, such as surface plasmon resonance [5], ellipsometry [6], and electrochemical methods [7, 8]. The electrochemical methods have many theoretical advantages such as fast operation, ease of use, inexpensiveness, portability, tolerance to sample turbidity, and often ultrasensitivity for identifying target analytes in complex biological samples [9, 10]. For instance, Domnanich et al. [11] immobilized antibodies electrostatically onto a xanthan/chitosan-polyelectrolyte-covered gold-chip microarray for detecting the melanoma-relevant marker protein S100B by fluorescence.

Melanoma cells originate from melanocytes, which are located near the basal layer of the epidermis [12]. Melanoma needs to be identified at early stages when it is still localized near the skin surface to enable curing by surgery before the penetration of melanoma cells from the upper layers of the skin reaches the bloodstream for spreading and the formation of metastases. Recently, the electrochemical detection of melanoma has been demonstrated in excised skin by addressing the enzyme tyrosinase (TYR), which is present in melanosomes and melanocytes as one part of the synthesis of the skin pigment melanin. Histopathologic TYR assays are based on TYR primary antibodies that are linked to secondary antibodies labeled with HRP. HRP can catalyze reactions to generate colored (for optical assays) or electro-active species (for electrochemical assays). Such assays for TYR show high sensitivity (>90\%) and specificity (>97\%) for primary melanoma [13]. They can also be electrochemically 
read-out by employing electro-active TYR substrates and derivatives, such as L-3,4dihydroxyphenylalanine (L-DOPA) [14]. Although histopathologic analysis of biopsied skin samples will remain the gold standard for confirming melanoma, there is also an interest in non-invasive and painless screening methods that support dermatologists in deciding whether a suspicious skin region should be excised for pathology or whether it can be inspected over a longer period without invasive intervention. Electrochemical sensing in this sense can be realized by using microneedle-type electrodes penetrating the epidermis. The microneedles must be coated with or made of a suitable electrode material that is non-toxic, stable, and provides a sensitive response. In vivo electrochemical detection of TYR requires further biocompatible needle electrode coatings, such as solid electrolytes or hydrogels, to provide a liquid electrolyte layer between the electrodes (including a counter and optionally a reference electrode) and the skin. This electrolyte layer must contain an electroactive species, which diffuses inside the electrolyte layer and can react with one or more analytes in the skin, such as with the protein TYR. TYR is an intracellular protein, and addressing it sufficiently might require permeabilizing or rupturing the cell membrane. This might be achievable directly by using the needles. So far, only a few works have been published on wearable TYR electrochemical sensors for the screening of melanoma on the skin, from which one example is the work of Wang and co-workers [3].

Herein, the fabrication and electrochemical characterization of nine gold-coated microneedles on one base as a working electrode with an external reference electrode and the counter electrode are presented. This work shows preliminary data, but the strategy could be further developed in the future and applied for in vivo skin measurements. The active electrode area at the cone of the gold needle was defined by insulating the base part of the MNs with a photocurable polymer. An ex vivo study has been done with dummy skin composed of an agarose gel with controllable concentrations and distribution patterns of TYR. The gold MNs were coated with an alginate-based hydrogel that was loaded with an electrolyte and catechol. TYR catalyzes the oxidation of catechol in the presence of oxygen, and the main product of the reaction, that is, o-benzoquinone, is electrochemically detectable at the gold electrodes. This concept principally enables the use of electrochemical catechol redox cycling as a measure for the presence and expression of TYR in the immediate environment of the MN. The microneedle chip was mounted to the z-motor of an SECM to enable the precise penetration of the MNs into a dummy skin. This allows the recording of the lateral and vertical distribution of TYR, which is essential for the electrochemical identification of the progression stage of melanoma and for studying the MN performance in general. 


\section{Results and discussion}

\subsection{Fabrication of the polymeric base of MNs}

Fig.1 shows the steps of fabrication of microneedle. The microneedle (MN) chip was fabricated from a stainless steel MN template as a positive mold (Fig.1(1-3)). This stainless steel $\mathrm{MN}$ template was fabricated by using electrical discharge machining, which uses electrical discharges (sparks) generated between a workpiece (anode) and a shaping piece (anode). Material is anodically removed from the workpiece in a three-dimensional manner by a series of rapidly recurring current discharges between the two electrodes. The gap between the two is filled with a dielectric liquid due to an applied electric voltage [15]. Fig.S1(a-d) shows the different steps of electrical discharge machining to fabricate the stainless steel MN of the machine. A very thin copper wire (Fig.S1a) is guided near to the stainless steel block (i.e., the workpiece) into which the MNs were to be fabricated (Fig.S1b). The block stand on the holder and the wire were translated relative to each other in the directions shown in Fig.S1e. The result was a three-dimensional steel template containing nine needles in the shape of equal pyramides (Fig.S1f). To get the pyramid-shaped needles, the electrical discharge machining process was carried out twice with intermediate rotation of the workpiece by $90^{\circ}$.

(1)

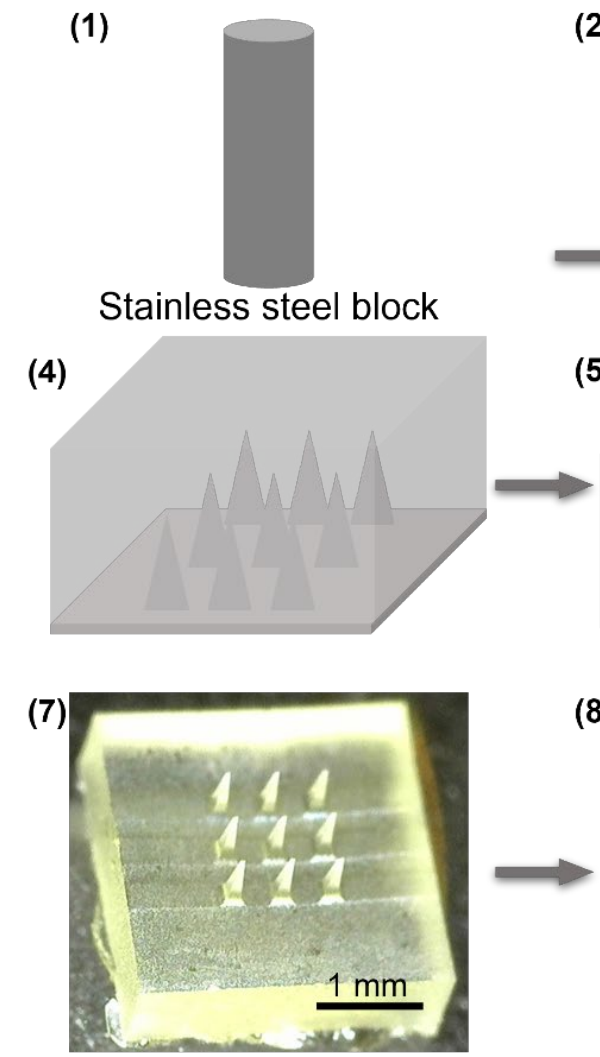

EMD6415 MN
(2)

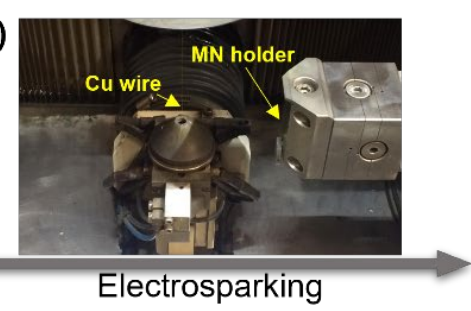

(5)

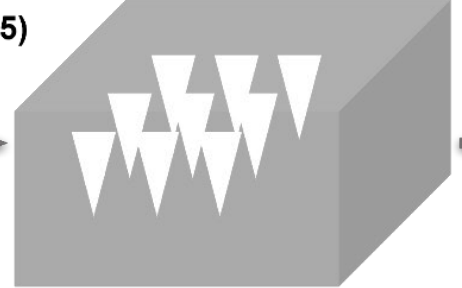

(8)

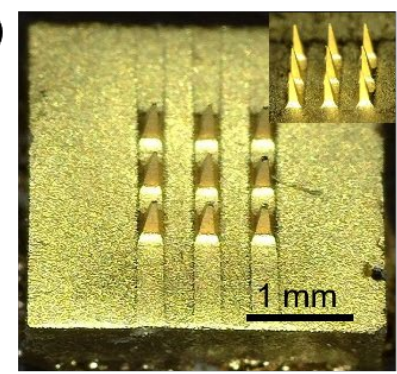

(3)

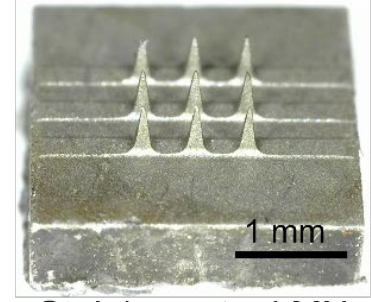

Stainless steel MN

(6)

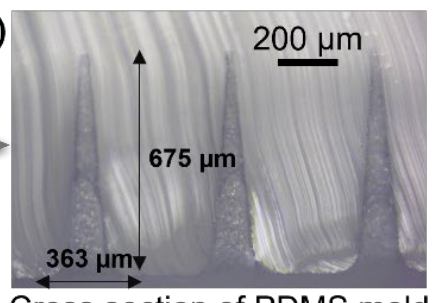

Cross section of PDMS mold

Fig.1 Steps of microneedle fabrication: (1) Stainless steel block. (2) Electrosparking. (3) Macroscopic image of stainless steel microneedle. (4) PDMS molding of stailess steel microneedle. (5) PDMS mold. (6) Laser scanning image of cross section of PDMS mold. (7) Macroscopic image of EMD64 microneedle. (8) Gold coated microneedle. (9) Laser scanning microscopy mage of gold needles. 
Laser scanning microscopy (LSM) was used to characterize the dimensions of the microneedles. LSM images of one example of the as-obtained stainless steel microneedle template are shown in Fig.S2. Four side views of the MN template are presented. As shown in Fig.S2, the MNs have the same shape and size on all four sides. The MNs have further a height of $\sim 675 \mu \mathrm{m}$ and a base width of $\sim 363 \mu \mathrm{m}$, which are dimensions suitable for insertion into the skin, in which the epidermis contains the proteins of interest this work, has a thickness of up to $700 \mu \mathrm{m}$. However, the applied MN template fabrication method is flexible in terms of the design of the MN template so that geometry adjustments are facile by modifying the digital process parameters. After that, a polydimethylsiloxane (PDMS) negative mold of the MNs was made from the stainless steel template (Fig.1(4-6)). The mold's replicate was created by casting the PDMS precursor mixture with a 10:1 weight ratio of elastomer to curing agent. The PDMS precursor mixture was degassed and subsequently cured in an oven at $80{ }^{\circ} \mathrm{C}$ for $4 \mathrm{~h}$ to generate the negative mold of the stainless steel master. The solid PDMS negative mold was detached from the steel template and, afterward, its surface was treated with oxygen plasma to make the surface hydrophilic. This will enable an easier removable of the pieces fabricated after that. The cross-section of a PDMS negative mold is shown in Fig.S3. The PDMS negative mold contained MN negatives of a depth of $\sim 650 \mu \mathrm{m}$ and a maximum width of $\sim 278 \mu \mathrm{m}$. PDMS molds are suitable for casting many kinds of polymers. The mechanical strength of MNs is critical to insert them into matrixes like skin successfully. Therefore, the casting polymer for MN production was done based on commercial methacrylate-based UV photopolymerizable ink (EMD6415 from SunChemical) (Fig.1(7)). The procedure for the fabrication of the EMD6415-based MN chip was rather facile. The UV-photo-curable ink was poured into the PDMS mold and cured under UV light irradiation for 48 hours. A solid and rigid polymer was obtained after 4 hours. It is known that methacrylates are rather toxic. Therefore, it must be guaranteed that the polymerization was complete before using the MN chips further. Indeed, the ink was photochemically cured until the yellowish color of the initial EMD6415 ink changed to nearly completely transparent. Fig.S4 shows a microscopic image of an MN chip based on the EMD6415-based polymer. Furthermore, the MNs were characterized by scanning electron microscopy (SEM, Fig.S5). The tip of the MN contained a quasi-plateau with a length of about $8 \mu \mathrm{m}$ and a width of about $500 \mathrm{~nm}$. Ideally, a sharp tip would have been desired, but the accuracy of the template fabrication was limited. A gold layer was deposited as a conductive electrode layer onto the polymeric EMD6415 MN base plate by thermally subliming gold particles at around $0.185 \mathrm{~A} / \mathrm{s}$ (1 hour evaporation procedure) using a DP650 sputtering machine (Alliance-Concept) (Fig.1(8, 9)). Fig.S6 shows a microscopic photo of an Au-coated MN electrode (a) and an LSM of the cross-section of a single microneedle (b). Fig.S7 shows the XRD pattern of the thin gold layer on the EMD6415 MN chip. Gold nanocrystals exhibited four distinct peaks at $2 \theta=38.1,44.3,64.5$, and 77.7 . All the four peaks 
corresponded to standard Bragg reflections (111), (200), (220), and (311) of face center cubic (fcc) lattice [16], indicating the successful gold coating on the EMD6415 MN base plate.

\subsection{Mechanical behavior of gold-coated MNs}

An essential challenge for using MNs is to effectively and reproducibly penetrate the MNs into the skin. For establishing designs allowing effective and repeatable MN punctures, a good understanding of the mechanical characteristics of the MNs is essential. The mechanical behavior of the MNs was evaluated by gently applying a force from the top of the needles until they fail, i.e., their breaking. The force loading system for assessing the mechanical properties of the MNs is shown schematically in Fig.S8. Fig.2 shows the mechanical behavior of the gold-coated EMD6415 MN chip. Fig.2a shows the load-displacement curves recorded with the speed of $0.05 \mathrm{~mm} . \mathrm{s}^{-1}$. The failure of the microneedle chip occurred at $0.22 \mathrm{~mm}$ with an applied force of $0.7 \mathrm{~N}$. The results show that the MNs showed a 2.5-3 times higher strength than many other values reported in the literature [17]. The stress-strain curve of the MN chip was also plotted, in which the stress is defined as force per area of the applied force. The total area of the MN chip was $1 \mathrm{~mm}^{2}$, as shown in Fig.2b. The mechanical strain is defined as the displacement per the whole length, which for the microneedles on average is $675 \mu \mathrm{m}$. Fig.2c shows the stress-strain plot of the EMD6415 MN chip. The Young's Modulus data collected in this study at low levels of deformation in the elastic part is shown in Fig.2d. The elasticity of the $\mathrm{MN}$ is $0.78 \mathrm{~N} \cdot \mathrm{mm}^{-2}$, which demonstrates a value that is suitable for initial insertion into a material like skin [18].

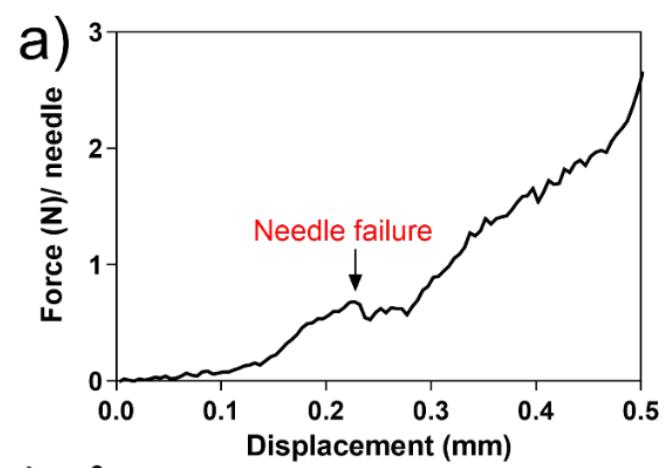

b)
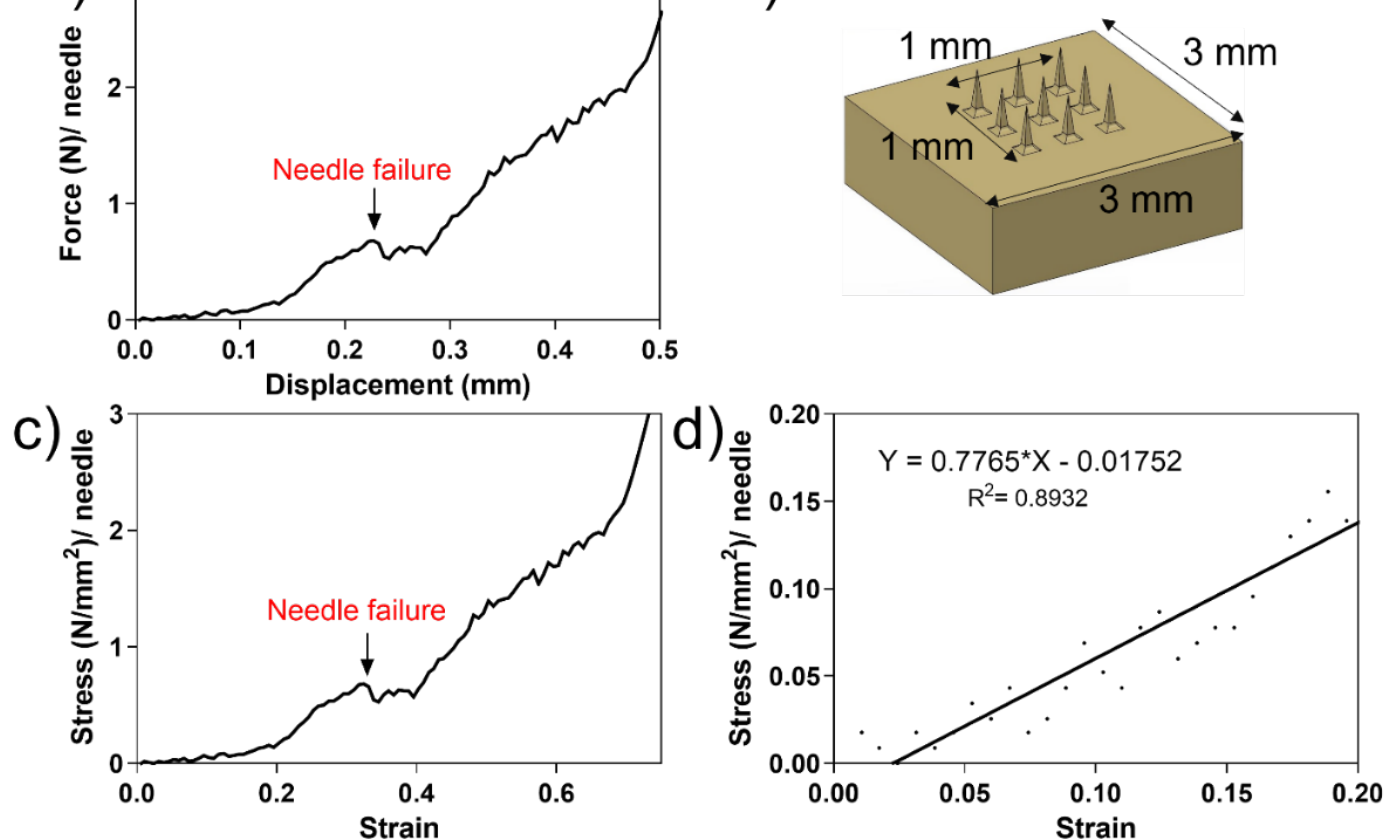

Fig.2 Analysis of the mechanical strength of the EMD6415 MN chip. (a) Load- displacement plot. (b) The scheme of the top view of MN shows the dimensions used for calculations for creating Fig.2c and Fig.2d. (c) The stressstrain plot, and (d) the stress-strain plot with a zoom into the elastic part. 


\subsection{Hydrogel coating of the microneedles}

For electrochemical measurements in the skin, the MN electrodes must be coated with a solid or highly viscous, stable electrolyte layer. Herein, a calcium alginate hydrogel was deposited on the needles after cross-linking sodium alginate with $\mathrm{Ca}^{2+}$ (added as $\mathrm{CaCl}_{2}$ ) in the presence of glycol according to the scheme shown in Fig.3a. The base of the gold MN chip was insulated using the UV-curable dielectric ink EMD6415, as shown in Fig.S9.

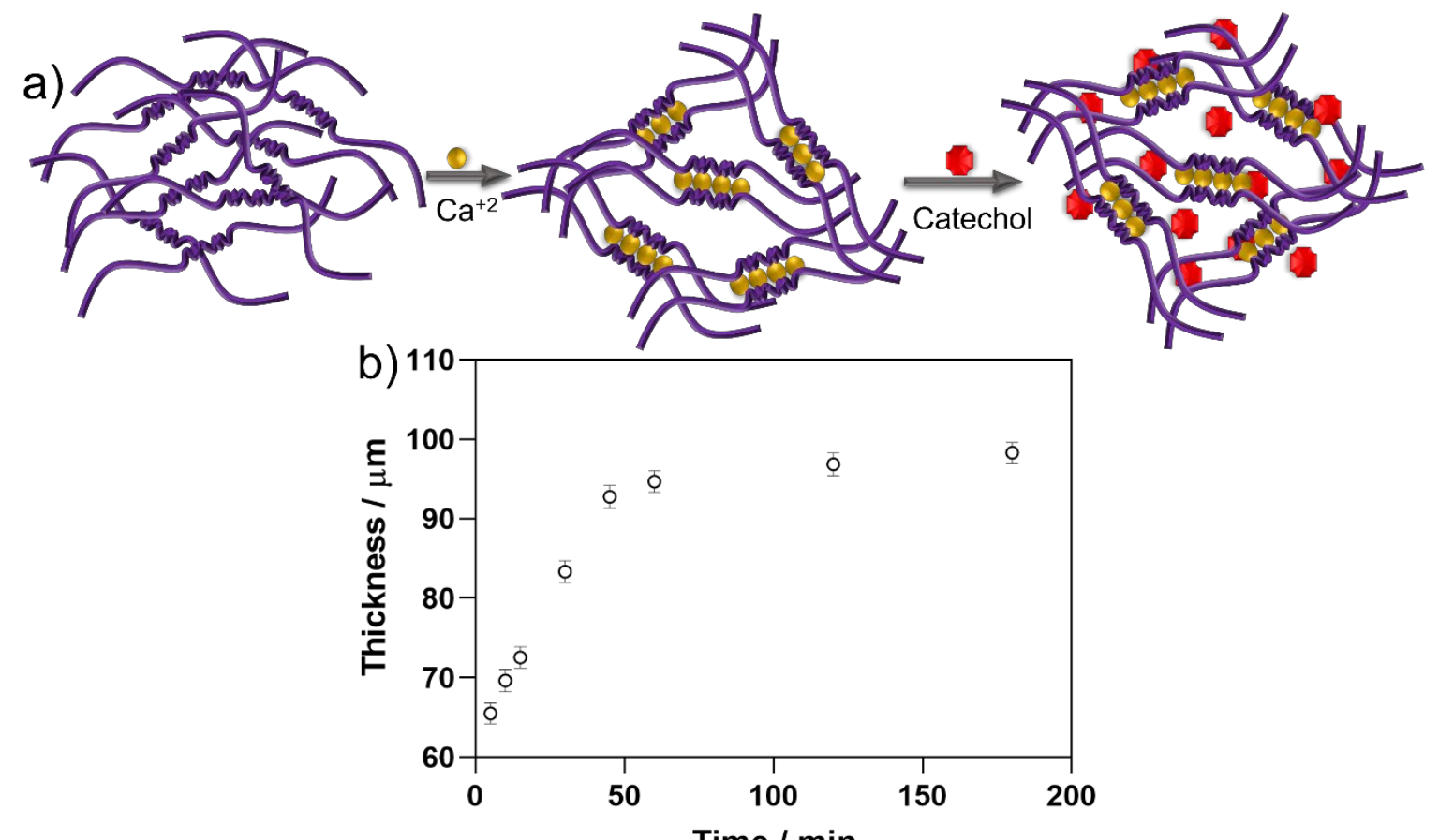

Fig.3 (a) Schematic representation of alginate hydrogel formation and subsequent loading with catechol. (b) Thickness of CaAlg/glycerol hydrogel layer on gold as a function of immersion time.

The deposition of the hydrogel on the MNs was realized by dip-coating the MNs into the freshly-prepared hydrogel. After that, the gel-coated MNs were immersed in a solution containing catechol to load the sensor with that electro-active compound. The thickness of Caalginate/glycerol hydrogels on the sensor was estimated for different times of dip-coating (5 min - $180 \mathrm{~min}$ ) using a flat gold-coated sample and measuring the hydrogel thickness with a laser microscope. The plot in Fig.3b shows the thickness obtained by dip-coating as a function of the dipping time. As shown, after 50 min the thickness of the hydrogel coating approached a plateau, and after about $1 \mathrm{hr}$ the thickness of hydrogel reached $90 \mu \mathrm{m}$. It was assumed that the thickness of the hydrogel coating on the microneedle surface was similar.

\subsection{Electrochemical properties of hydrogel coated gold MNs}

Electrochemical measurements have been carried out by mounting an MN chip holder, produced in-house, to the z motor of the Märzhäuser SECM system (Fig.4(a-c)). One needle plate was used as WE (consisting of nine electrically connected Au MNs), one needle plate of $\mathrm{Au} \mathrm{MN}$ as $\mathrm{CE}$, and one needle plate of $\mathrm{Ag} \mathrm{MN}$ as a QRE. First, cyclic voltammetry 
measurements were performed for the bare, alginate, and catechol/alginate coated gold MN electrode, from which some examples are shown in (Fig.4d). The sensor was immersed in PBS that contained in all cases FcMeOH. The redox peaks for FcMeOH are clearly observed in all cases. Comparing the response of the gold MN electrode with and without hydrogel, it can be clearly seen that the presence of the alginate gel reduced the active area of the WE as the redox peaks for the oxidation and reduction of $\mathrm{FCMeOH}$ were substantially reduced. However, this result demonstrates that a redox species like $\mathrm{FcMeOH}$ diffused from the sample solution through the alginate gel to the MN electrode. When the gel had been loaded with catechol, the redox peaks in the CV were increased, most likely due to the presence of the two redox-active species (FcMeOH plus catechol). A CV using the catechol-loaded alginatecoated MN chip immersed in PBS is shown in Fig.4e. The typical redox peaks for catechol can be seen demonstrating the general functionality of the hydrogel as an electrolyte layer. In all of the cases, the supporting electrolyte was PBS. In order to investigate the sensitivity of the sensor for TYR, first solutions with different concentrations of TYR in PBS were prepared, and the catechol-loaded hydrogel MN chip was inserted. The detection principle was the following: the oxidation of catechol was catalyzed by TYR, and the as-generated obenzoquinone was electrochemically reduced at the Au-MN electrode. The concept is based on the assumption that the higher the TYR concentration in the environment of the gel-coated MN chip, the higher the reduction current of o-benzoquinone. The sensitivity for TYR of the sensor was determined as $47.04 \mu \mathrm{A} \cdot \mathrm{mg}^{-1} \cdot \mathrm{mL}$ (Fig.4f). To obtain this value, chronoamperometry was carried out in an unstirred solution, and the current values at $100 \mathrm{~s}$ were taken. 

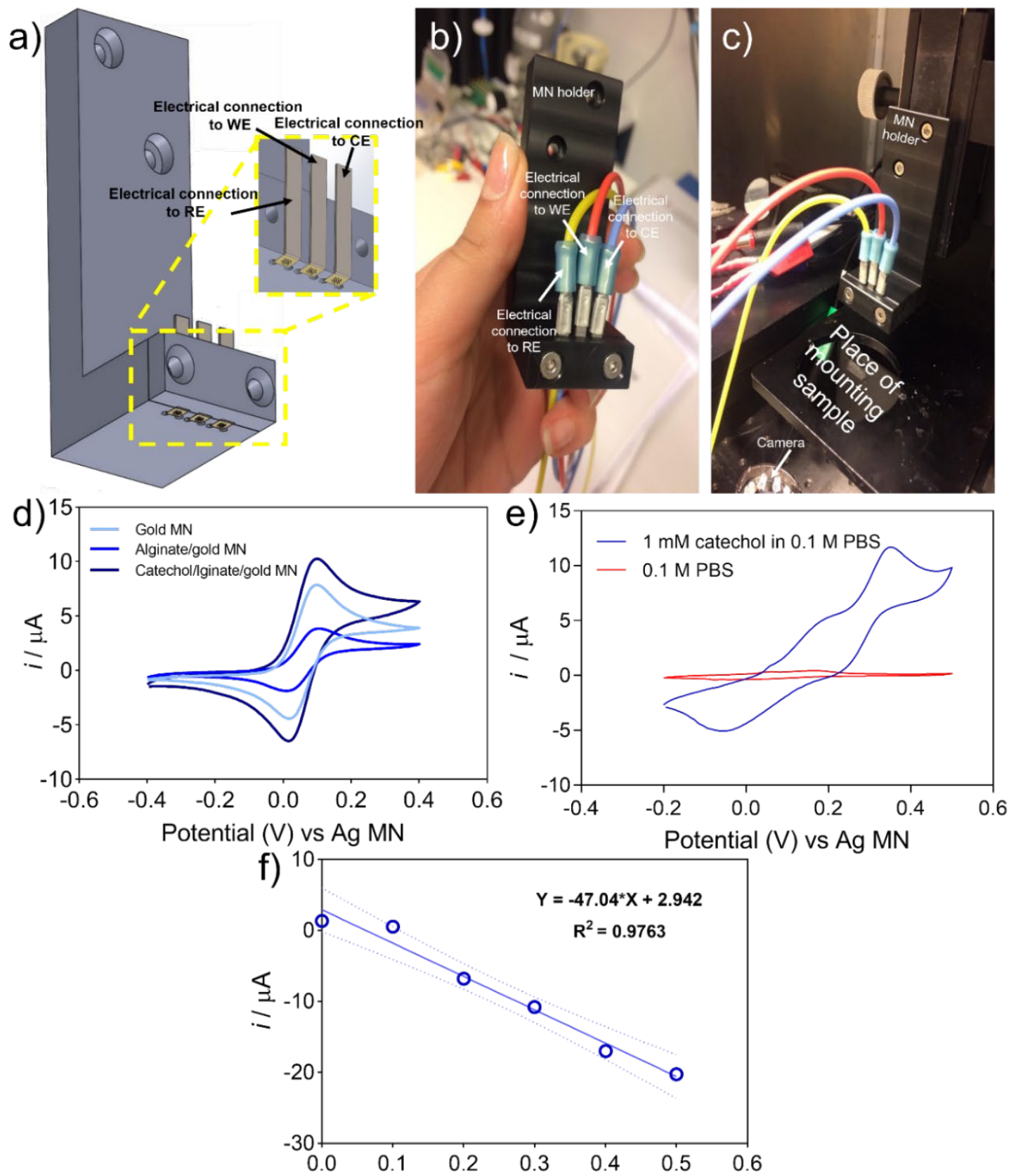

Concentration of TYR in agarose gel / $\mathrm{mg}_{\mathrm{mL}} \mathrm{mL}^{-1}$

Fig.4 (a) Schematic representation of the three-electrode setup connected to the z-motor of an SECM. (b) Photograph of the holder. (c) Photograph of the holder mounted to the z-motor. (d) Cyclic voltammogram of Gold MN, Alginate/gold MN, and Catechol@alginate: gold MN biosensors in $5 \mathrm{mM} \mathrm{FCMeOH}$ in $0.1 \mathrm{M}$ PBS. Scan rate= $50 \mathrm{mV} / \mathrm{s}$. (e) Cyclic voltammogram of one exemplary alginate-coated gold microneedle electrode in $1 \mathrm{mM}$ catechol in $0.1 \mathrm{M}$ PBS and pure $0.1 \mathrm{M}$ PBS buffer. Scan rate= $50 \mathrm{mV} / \mathrm{s}$. (f) Calibration curve extracted from chronoamperometry curves of Catechol@alginate: gold MN biosensor for different concentrations of TYR in PBS. Applied potential $=-0.115 \mathrm{~V}$, time $=100 \mathrm{~s}$.

The final measurements of this part were done in dummy skin made of an agarose gel (Fig.5a). TYR of different concentrations was injected locally into the dummy skin generating a simplified model with TYR-containing features. Fig.5b demonstrates the electrochemical detection principle that was discussed vide supra. Fig.5(c,d) shows the electrochemical behaviour of the catechol-loaded gel MN electrodes that were fully inserted into the dummy skin to detect TYR. Compared to the sensor response in the solution, the concentration of 
TYR in the gel is an assumption due to the practical difficulties in preparing a skin model accurately. The sensitivity for TYR of the MN sensor in dummy skin, obtained from reading the $\mathrm{MN}$ reduction current at 100 s with an applied potential of $-0.115 \mathrm{~V}$, was about $7.52 \mu \mathrm{A} \cdot \mathrm{mg}^{-}$ ${ }^{1} . \mathrm{mL}$. This relatively long time of 100 s was selected as the current signals reached quasiplateaus.

a)

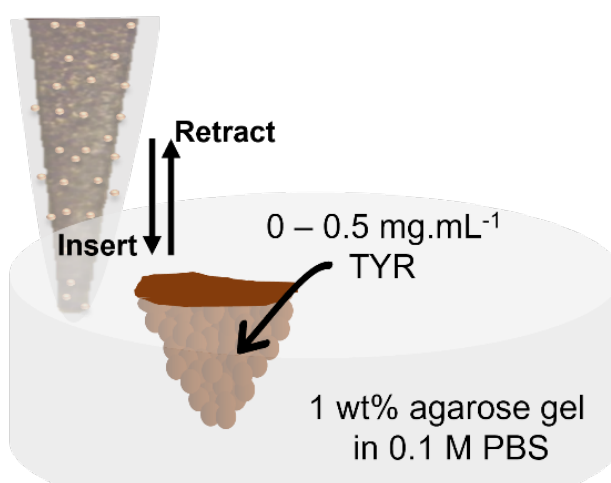

C)

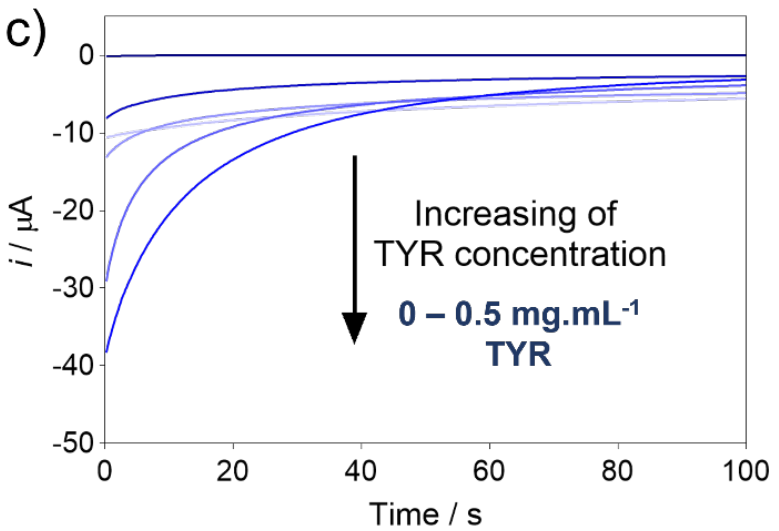

b)
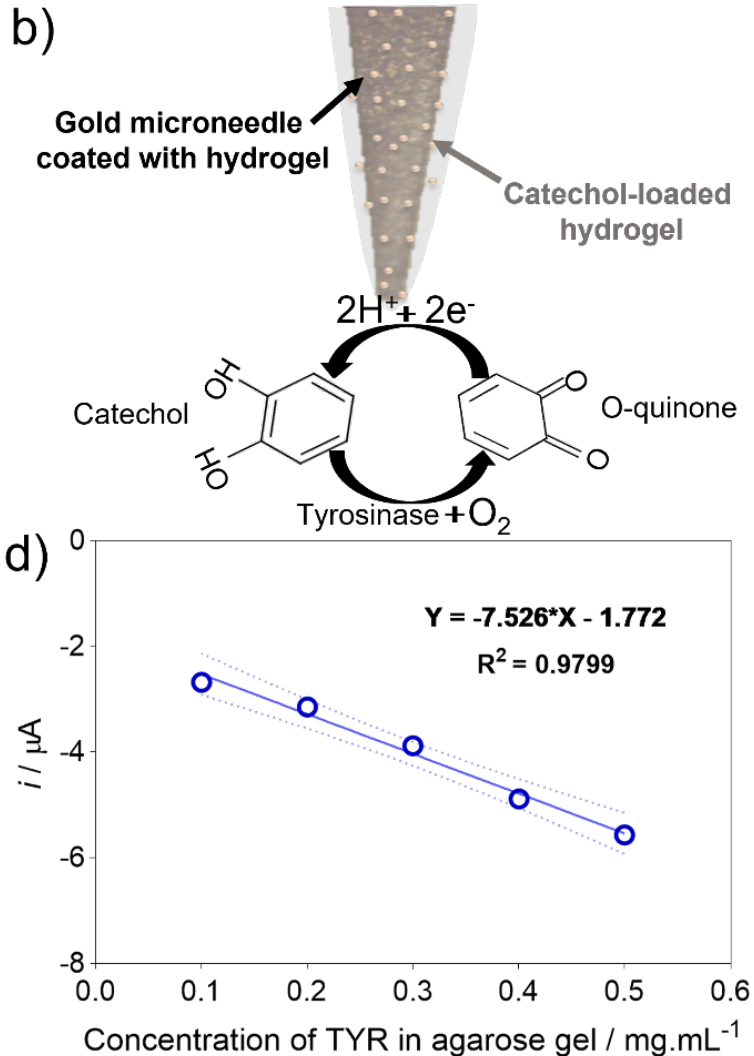

Fig.5 (a) Schematic representing the electrochemical readout strategy of the gel-based MN sensor for TYR. (b) The Schematic represents the detection principle for TYR based on the recycling of catechol. (c) Electrochemical detection of TYR using chronoamperometry of $0.1-0.5 \mathrm{mg} / \mathrm{mL}$ TYR in agarose gel. (d) Calibration curve extracted from chronoamperometry curves (c). Applied potential $=-0.115 \mathrm{~V}$, time $=100 \mathrm{~s}$.

Selectivity is an important factor in analytical measurements to determine the performance of a designed sensor. Catechol@alginate: gold MN shows a low negative potential of about $0.115 \mathrm{~V}$ vs. $\mathrm{Ag} / \mathrm{AgCl}$ for the detection of benzoquinone, and the probability of catechol oxidation with a variety of components in skin or sweat is low. Several electroactive skin components, including glucose [19], ascorbic acid [20], uric acid [21], histidine [22], cysteamine [23], and glutathione [24], were examined by inserting $1 \mathrm{mM}$ of each component in the dummy skin. Although, as most of these compounds are reducing agents, they are not suspected of oxidizing catechol, however, they could reduce o-benzoquinone. The current signals in Fig. 6 in the bare 1 wt\% agarose (black line, "blank") and in the presence of other constitutes such as $1 \mathrm{mM}$ glutathione (Fig.6a), $1 \mathrm{mM}$ uric acid (Fig.6b), $1 \mathrm{mM}$ glucose (Fig.6c), $1 \mathrm{mM}$ ascorbic acid (Fig.6d), $1 \mathrm{mM}$ histidine (Fig.6e), $1 \mathrm{mM}$ cysteamine (Fig.6f), and $1 \mathrm{mg} \cdot \mathrm{mL}^{-1}$ TYR (Fig.6g) were evaluated. The current signal in the presence of TYR 
demonstrated a clear difference compared to all other components. The relative current values corresponding to different compounds in dummy skin are shown in Fig.6h. Such differences in the responses between TYR and other components suggest a high selectivity of the MN sensor toward TYR screening, however not considering that competing compounds in the skin could reduce o-benzoquinone and thus compete with the electrode for this electro-active species.
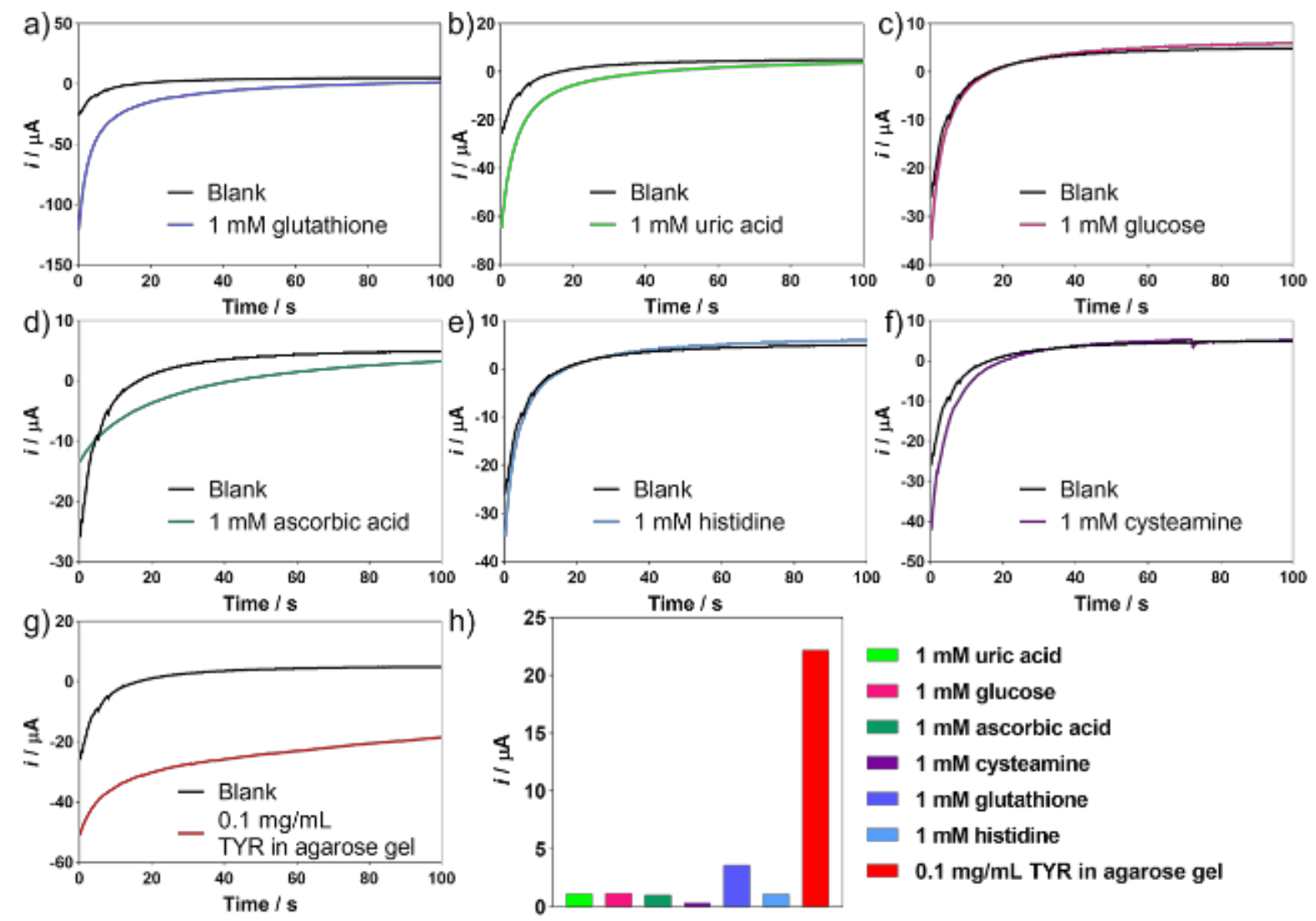

Fig. 6 Sensor selectivity in the presence of potential interferences. Amperometric response of the microneedle sensor before (black) and after (red) contact with dummy skin, (a) $1 \mathrm{mM}$ glutathione, (b) $1 \mathrm{mM}$ uric acid, (c) $1 \mathrm{mM}$ glucose, (d) $1 \mathrm{mM}$ ascorbic acid, (e) $1 \mathrm{mM}$ histidine, (f) $1 \mathrm{mM}$ cysteamine, (g) $0.1 \mathrm{mg} / \mathrm{mL}$ TYR. (h) Relative current values corresponding to different compounds in phantom tissue $(\mathrm{a}-\mathrm{g})$. Applied potential $=-0.115 \mathrm{~V}$, time $=100 \mathrm{~s}$.

To investigate the reproducibility and stability of catechol@alginate: gold MN biosensors, five continuous measurements for the detection of o-benzoquinone were carried out in $1 \mathrm{wt} \%$ agarose gel containing $0.1 \mathrm{mg} \cdot \mathrm{mL}^{-1}$ TYR. Differential pulse voltammetry (DPV) was applied from $0.1 \mathrm{~V}$ to $-0.8 \mathrm{~V}$. The reduction of o-benzoquinone, which was produced by the oxidation of catechol at the electrode as well as by TYR, was carried out at the Au MN electrode leading to a reduction peak at $-0.15 \mathrm{~V}$. A relative standard deviation (RSD) was calculated by dividing the standard deviation by the average of currents. An RSD of $8.5 \%$ ( $n=5$ measurements with the same electrode) confirmed that the catechol@alginate: gold MN shows a reproducible performance for continuous TYR detection (Fig.7(a,b)). With experiment number, the current increased, which could probably be linked to slight continuous degradation of the hydrogel increasing the active $\mathrm{Au}$ electrode surfaces. In another set of experiments, five 
catechol@alginate: gold MN electrodes were fabricated and measured. An RSD of 3.95 \% $(n=5$ electrodes) was obtained (Fig.7(c,d)). These results suggest that the electrode fabrication process was reproducible, and Catechol@alginate: gold MN biosensors demonstrated high stability for the repetitive detection of TYR.
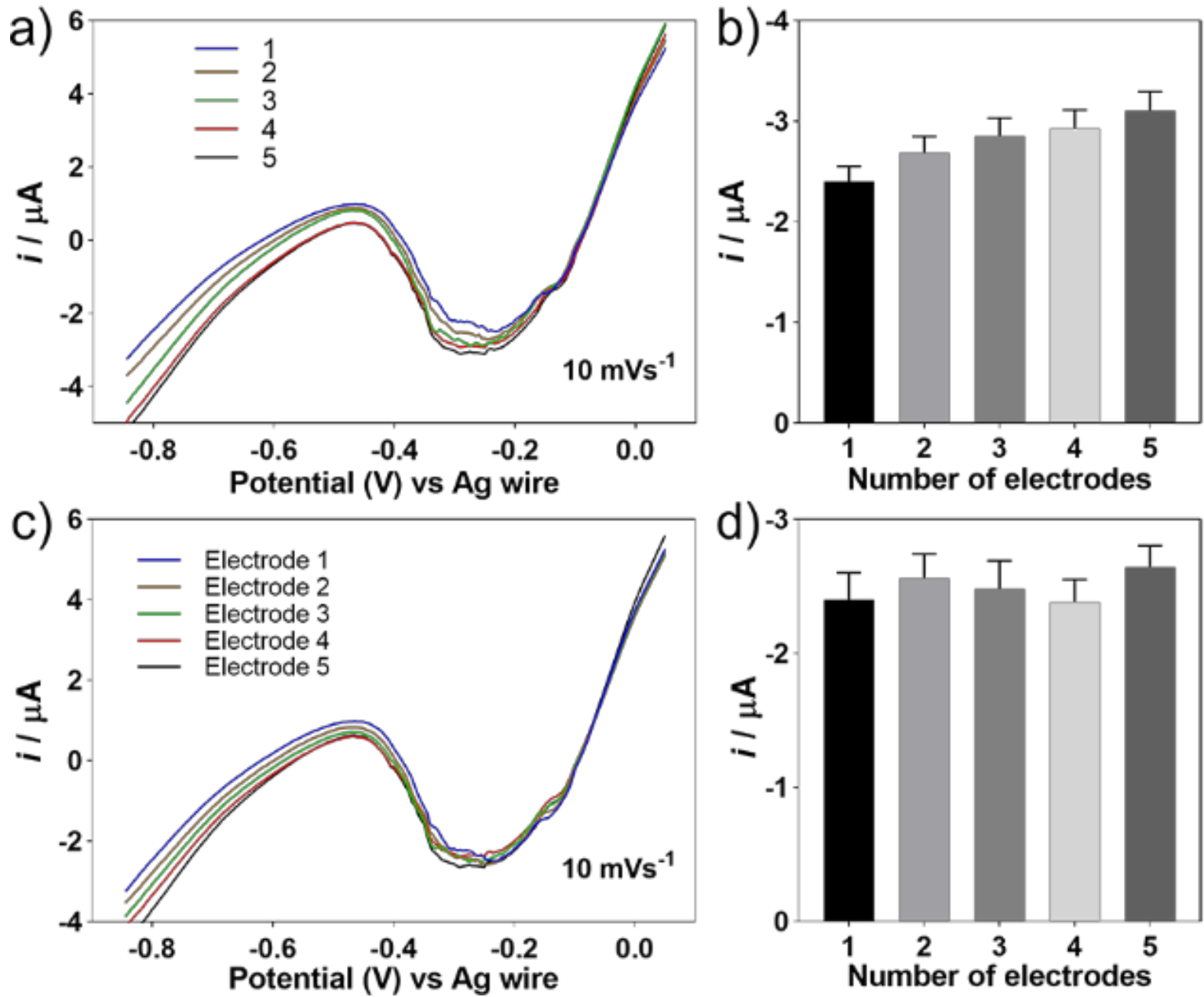

Fig.7 (a) and (b) Current response of Catechol@alginate: gold MN for detecting $0.1 \mathrm{mg} / \mathrm{mL}$ of TYR with five continuous measurements using the same electrode. (c) and (d) Current response of Catechol@alginate: gold MN for detecting $0.1 \mathrm{mg} / \mathrm{mL}$ of TYR with five identically made electrodes. The scan rate $=10 \mathrm{mV} / \mathrm{s}$, and the scan is from $-0.8 \mathrm{~V}$ to $0.1 \mathrm{~V}$.

\section{Conclusion}

This study proposed a fabrication protocol of an MN electrode chip that could be used for the large-scale fabrication of transdermal TYR sensors. The preliminary results in dummy skin showed promising properties of the MNs for sensing the TYR expression present in the matrix where the MNs are inserted in dummy skin. The microneedles were fabricated via PDMS casting to fabricate a polymer needle support. After that, the plastic microneedles were coated with a gold film by sputtering to produce an Au MN electrode. The gold MNs were then covered with an alginate gel loaded with catechol and PBS to provide a liquid electrolyte layer that contained an electroactive species, which is sensitive to oxidation that TYR catalyzes. The sensor showed high sensitivity of $7.52 \mu \mathrm{A} \cdot \mathrm{mg}^{-1} \cdot \mathrm{mL}$ TYR in dummy skin using 
chronoamperometry (readout time 100s). A relative standard deviation (RSD) of $8.54 \%$ ( $=5$ measurements with the same electrode) confirmed that Catechol@alginate: gold MN is reproducible, allowing for continuous TYR detection. Also, five parallel Catechol@alginate: gold MN electrodes were fabricated using the same experimental setup and showed an RSD of $3.95 \%$ ( $n=5$ electrodes). These results suggest that the electrode fabrication process was reproducible, and Catechol@alginate: gold MN biosensors demonstrated high stability for repetitive detection of TYR. Furthermore, the sensor showed reasonable selectivity in the presence of potentially interfering compounds, here reducing agents were tested. Considering that this work was preliminary, it can be envisaged to continue this approach by a) addressing the MNs individually for electrochemical bioimaging and b) to fabricate a more robust solid electrolyte layer, for instance with an conductive polymer as catechol derivate to improve the sensing further and to approach in vivo sensing.

\section{References}

[1] H. Lee, T.K. Choi, Y.B. Lee, H.R. Cho, R. Ghaffari, L. Wang, et al., A graphene-based electrochemical device with thermoresponsive microneedles for diabetes monitoring and therapy, Nature nanotechnology, 11(2016) 566-72.

[2] P.M. Wang, M. Cornwell, M.R. Prausnitz, Minimally invasive extraction of dermal interstitial fluid for glucose monitoring using microneedles, Diabetes technology \& therapeutics, $7(2005)$ 131-41.

[3] B. Ciui, A. Martin, R.K. Mishra, B. Brunetti, T. Nakagawa, T.J. Dawkins, et al., Wearable wireless tyrosinase bandage and microneedle sensors: toward melanoma screening, Advanced healthcare materials, 7(2018) 1701264.

[4] P.R. Miller, R.J. Narayan, R. Polsky, Microneedle-based sensors for medical diagnosis, Journal of Materials Chemistry B, 4(2016) 1379-83.

[5] S.C. Jena, S. Shrivastava, S. Saxena, N. Kumar, S.K. Maiti, B.P. Mishra, et al., Surface plasmon resonance immunosensor for label-free detection of BIRC5 biomarker in spontaneously occurring canine mammary tumours, Scientific reports, 9(2019) 1-12.

[6] Y. Niu, T. Kang, G. Jin, Joint detection of tumor markers with imaging ellipsometry biosensor, Thin solid films, 571(2014) 453-62.

[7] S. Darvishi, H. Pick, T.-E. Lin, Y. Zhu, X. Li, P.-C. Ho, et al., Tape-stripping electrochemical detection of melanoma, Analytical chemistry, 91(2019) 12900-8.

[8] J. Okuno, K. Maehashi, K. Kerman, Y. Takamura, K. Matsumoto, E. Tamiya, Label-free immunosensor for prostate-specific antigen based on single-walled carbon nanotube arraymodified microelectrodes, Biosensors and Bioelectronics, 22(2007) 2377-81.

[9] N.J. Ronkainen, H.B. Halsall, W.R. Heineman, Electrochemical biosensors, Chemical Society Reviews, 39(2010) 1747-63.

[10] J. Li, S. Li, C.F. Yang, Electrochemical biosensors for cancer biomarker detection, Electroanalysis, 24(2012) 2213-29.

[11] P. Domnanich, D.B. Peña, C. Preininger, Xanthan/chitosan gold chip for metal enhanced protein biomarker detection, Biosensors and Bioelectronics, 26(2011) 2559-65.

[12] N. Maddodi, A. Jayanthy, V. Setaluri, Shining light on skin pigmentation: the darker and the brighter side of effects of UV radiation, Photochemistry and photobiology, 88(2012) 107582.

[13] D. Weinstein, J. Leininger, C. Hamby, B. Safai, Diagnostic and prognostic biomarkers in melanoma, The Journal of clinical and aesthetic dermatology, 7(2014) 13. 
[14] T.-E. Lin, F. Cortés-Salazar, A. Lesch, L. Qiao, A. Bondarenko, H.H. Girault, Multiple scanning electrochemical microscopy mapping of tyrosinase in micro-contact printed fruit samples on polyvinylidene fluoride membrane, Electrochimica Acta, 179(2015) 57-64.

[15] K. Ho, S. Newman, State of the art electrical discharge machining (EDM), International Journal of Machine Tools and Manufacture, 43(2003) 1287-300.

[16] S. Krishnamurthy, A. Esterle, N.C. Sharma, S.V. Sahi, Yucca-derived synthesis of gold nanomaterial and their catalytic potential, Nanoscale research letters, 9(2014) 627.

[17] R.B. Groves, S. Coulman, J.C. Birchall, S.L. Evans, Quantifying the mechanical properties of human skin to optimise future microneedle device design, Computer methods in biomechanics and biomedical engineering, 15(2012) 73-82.

[18] J.J. García-Guzmán, C. Pérez-Ràfols, M. Cuartero, G.A. Crespo, Microneedle based electrochemical (Bio) Sensing: Towards decentralized and continuous health status monitoring, TrAC Trends in Analytical Chemistry, (2020) 116148.

[19] W. Groenendaal, G. Von Basum, K.A. Schmidt, P.A. Hilbers, N.A. van Riel, Quantifying the composition of human skin for glucose sensor development, SAGE Publications Sage CA: Los Angeles, CA2010.

[20] S. Ravetti, C. Clemente, S. Brignone, L. Hergert, D. Allemandi, S. Palma, Ascorbic acid in skin health, Cosmetics, 6(2019) 58.

[21] G.-e. Rhie, M.H. Shin, J.Y. Seo, W.W. Choi, K.H. Cho, K.H. Kim, et al., Aging-and photoaging-dependent changes of enzymic and nonenzymic antioxidants in the epidermis and dermis of human skin in vivo, Journal of investigative dermatology, 117(2001) 1212-7.

[22] N.K. Gibbs, M. Norval, Urocanic acid in the skin: a mixed blessing?, Journal of Investigative Dermatology, 131(2011) 14-7.

[23] M. Besouw, L. Van den Heuvel, R. van Eijsden, I. Bongaers, L. Kluijtmans, M. Dewerchin, et al., Increased human dermal microvascular endothelial cell survival induced by cysteamine, Journal of inherited metabolic disease, 36(2013) 1073-7.

[24] M. Rinnerthaler, J. Bischof, M.K. Streubel, A. Trost, K. Richter, Oxidative stress in aging human skin, Biomolecules, 5(2015) 545-89. 


\title{
- Supplementary Information -
}

\section{Ex vivo performance evaluation of a transdermal gold injectable microneedle for tyrosinase sensing}

\author{
Sorour Darvishi, ${ }^{[a]}$ Hubert H. Girault, ${ }^{*}[a]$

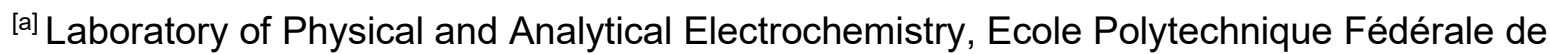 \\ Lausanne (EPFL) Valais Wallis, Rue de I'Industrie 17, 1950 Sion, Switzerland
}

\footnotetext{
* Corresponding author: Laboratory of Physical and Analytical Electrochemistry, Ecole Polytechnique Fédérale de Lausanne (EPFL) Valais Wallis, Rue de l'Industrie 17, 1950 Sion, Switzerland. Email addresses: hubert.girault@epfl.ch (H.H. Girault)
} 

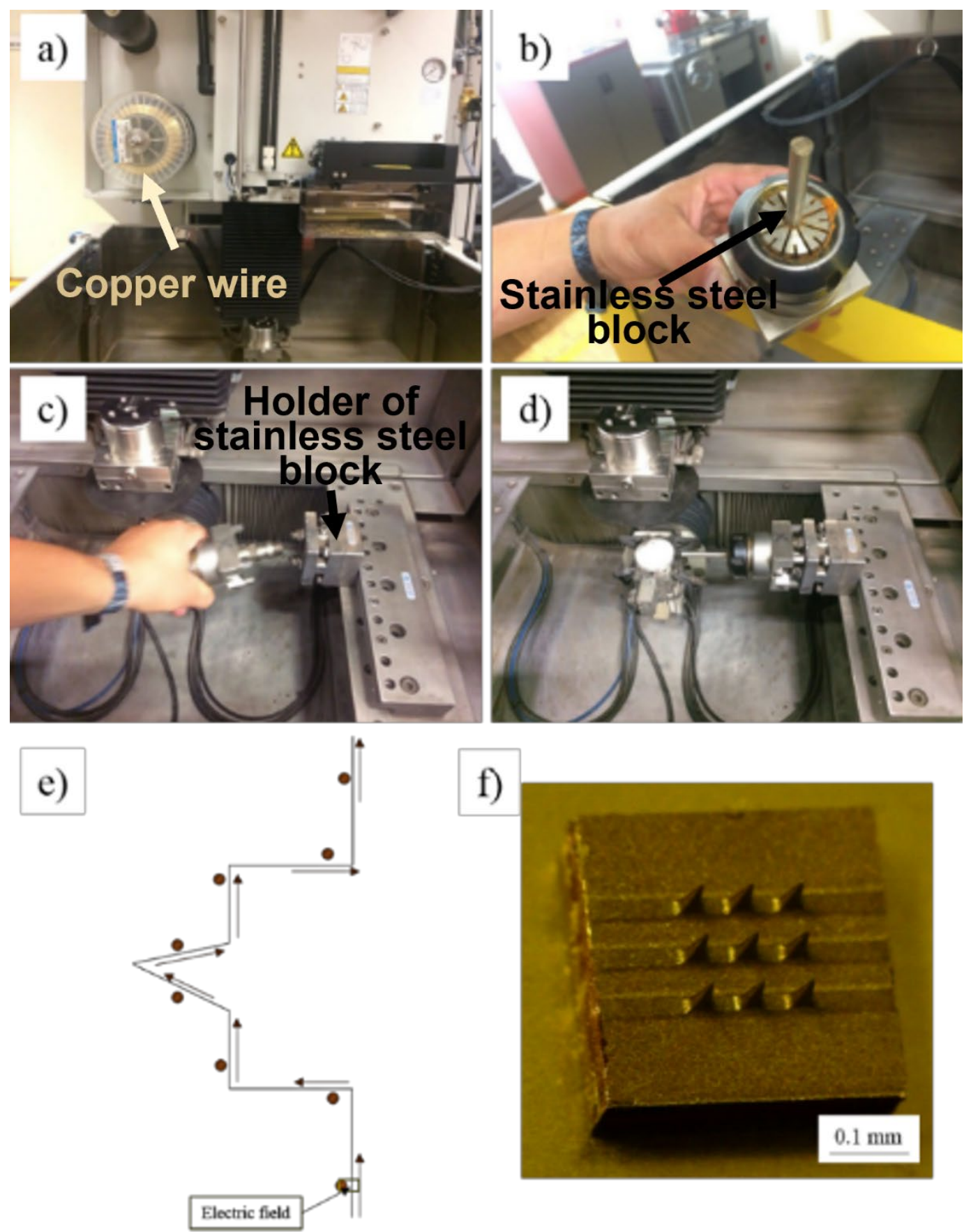

Fig.S1 (a) Copper wire for electrosparking. (b) Stainless steel block. (c) Holders of the stainless steel block. (d) The whole system of electrosparking. (e) The direction of movement of copper wire. (f) Stainless steel microneedle. 


\section{Dimension}

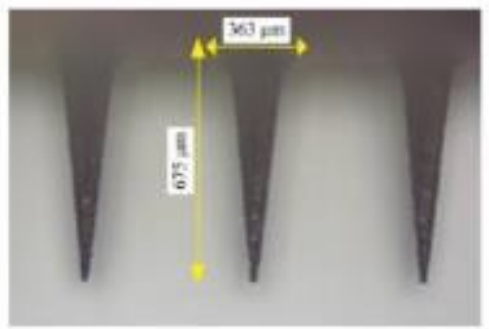

\section{Different view}

First side

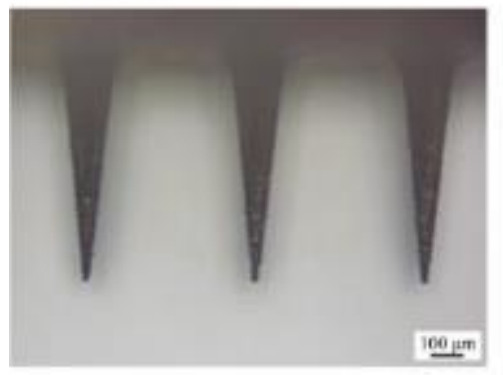

Third side

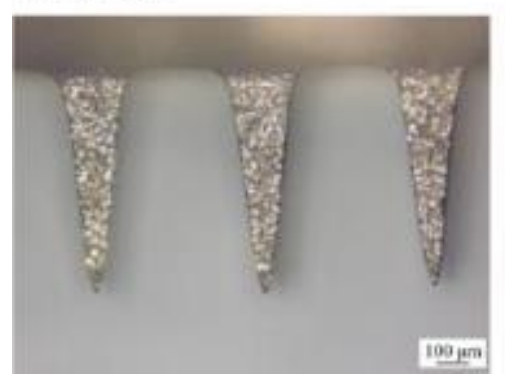

Second side

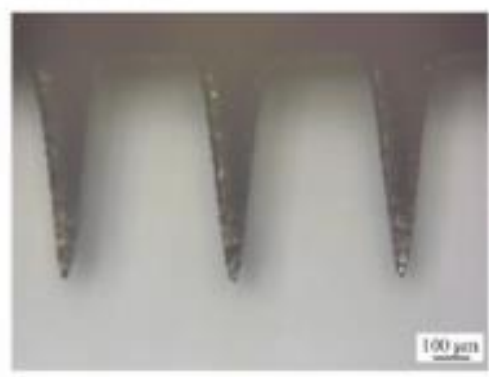

Forth side

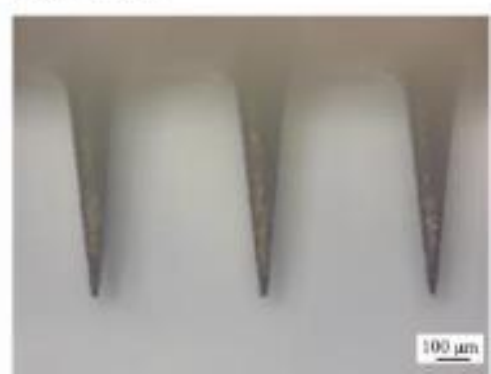

Fig.S2 Laser microscopy images of stainless steel MNs of the template from the four lateral sides of the template.
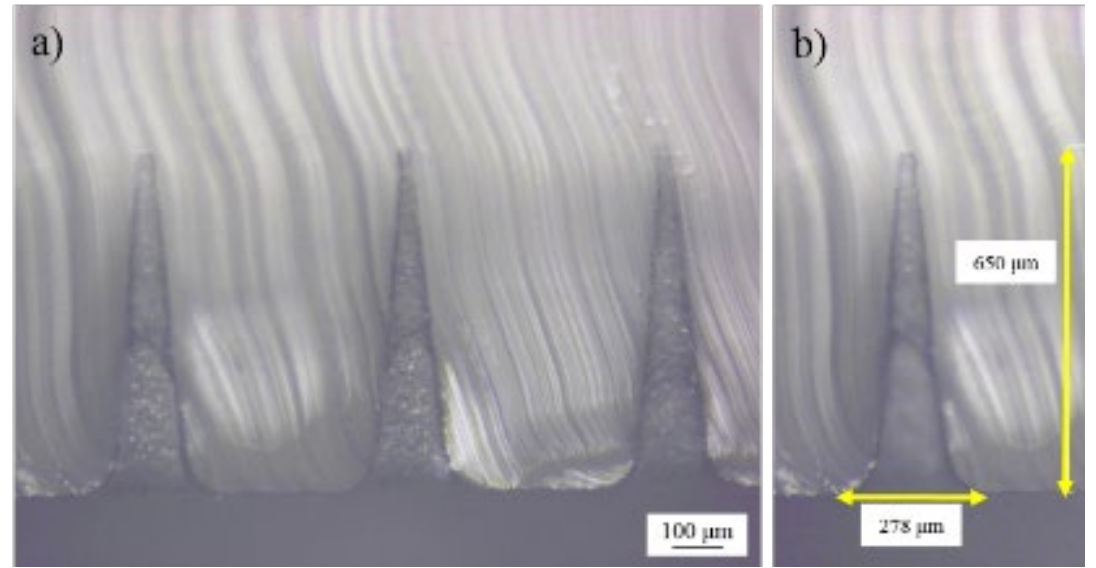

Fig.S3 Laser microscopy image of PDMS negative mold for the production of a MN chip; side view. 

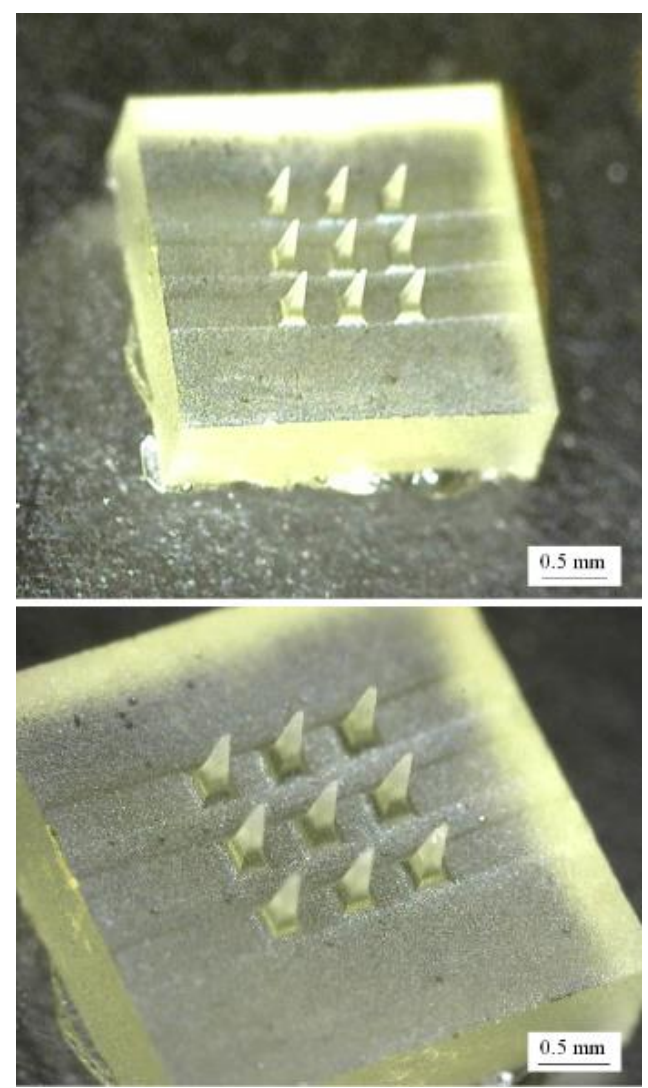

Fig.S4 Macroscopic image of an EMD6415-based MN chip.

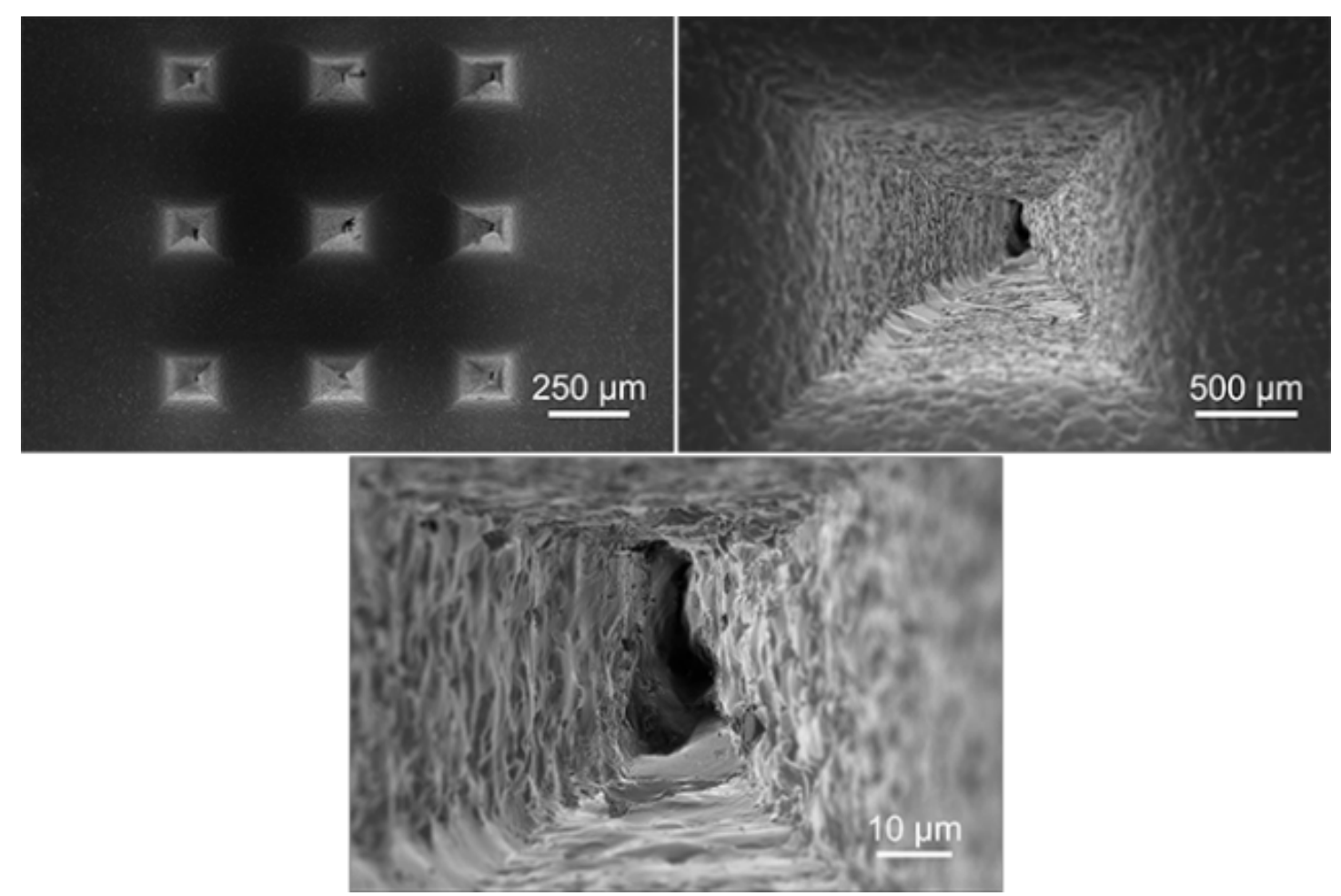

Fig.S5 SEM images of the EMD6415-based MN base plate (top views with different magnifications). 


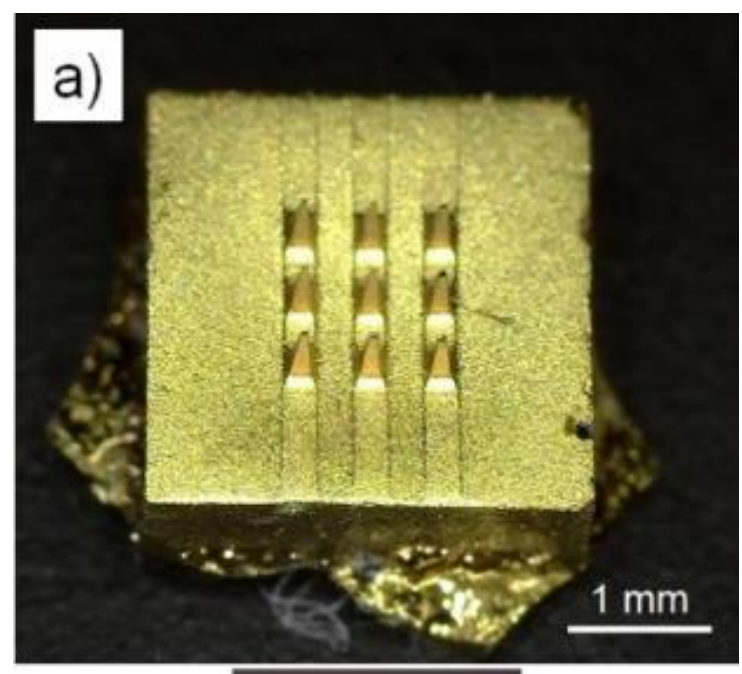

b)

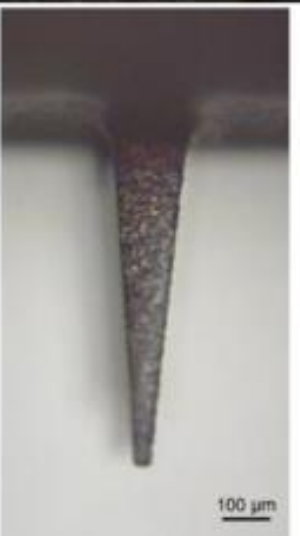

Fig.S6 (a) Microscopic image of a gold-coated MN chip. (b) Laser microscopy image of gold-coated MN (side

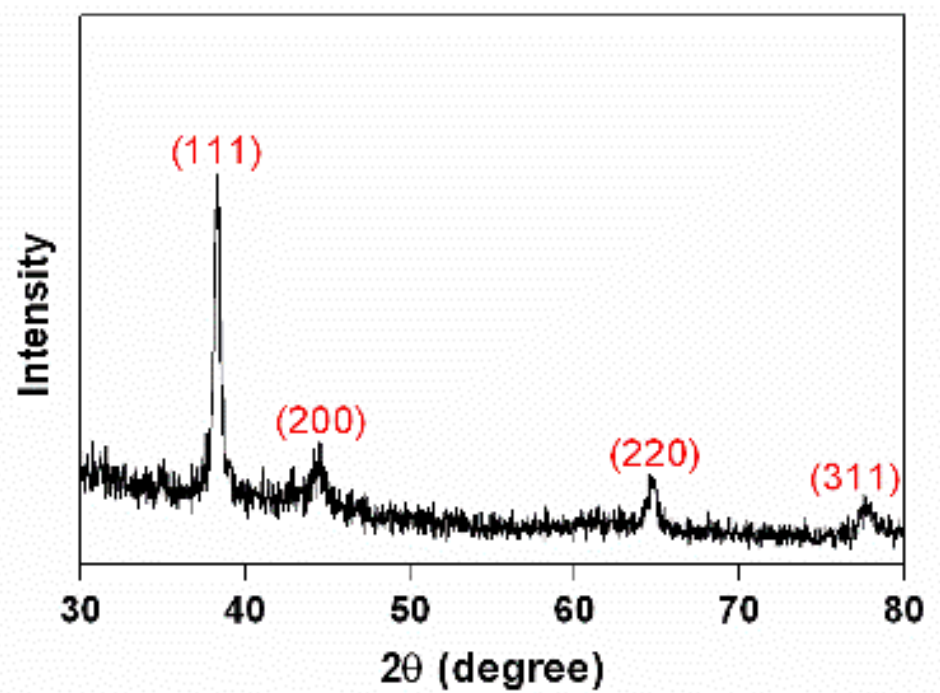

Fig.S7 XRD pattern of the gold layer on the MN chip. 


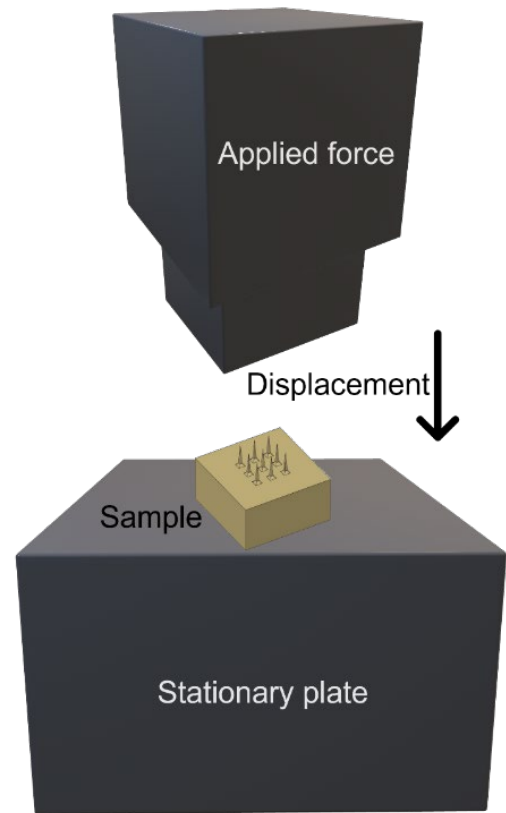

Fig.S8 Machine for characterization the mechanical behaviour of MNs.

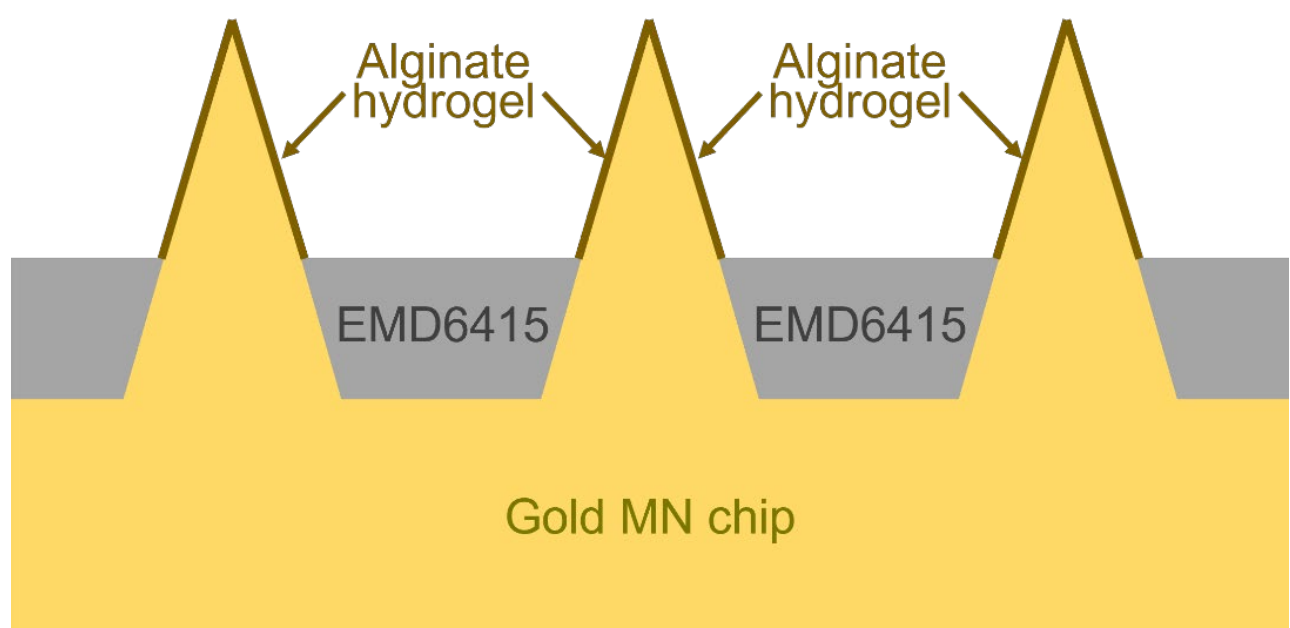

Fig.S9 Scheme illustrating all layers of the final MN sensor (cross-sectional view). 